\title{
Cambios de largo plazo en el paisaje y los ensambles de micromamíferos y rapaces en Chile central
}

\section{Long-term changes in landscape and in small mammal and raptor assemblages in central Chile}

\author{
EDUARDO F. PAVEZ ${ }^{1}$, GABRIEL A. LOBOS ${ }^{2} \&$ FABIAN M. JAKSIC ${ }^{2}$, * \\ ${ }^{1}$ Unión de Ornitólogos de Chile, Casilla 13.183, Santiago-21, Chile \\ ${ }^{2}$ Centro de Estudios Avanzados en Ecología \& Biodiversidad (CASEB), Pontificia Universidad Católica de Chile, \\ Santiago, Chile \\ * Autor correspondiente: fjaksic@bio.puc.cl
}

\begin{abstract}
RESUMEN
San Carlos de Apoquindo, en la precordillera de Chile central, es una localidad con estudios de larga data en vertebrados (ca. 30 años), situación favorable para el análisis de procesos de largo plazo que operan sobre dichos ensambles. En este contexto estudiamos la trayectoria del uso del suelo mediante imágenes satelitales LANDSAT, correspondientes a 1975, 1989 y 2003 y su presunto efecto sobre la vegetación, micromamíferos y aves rapaces. El análisis de las imágenes satelitales revela una expansión acelerada del área urbana en desmedro del suelo agrícola y matorrales naturales. Las especies de micromamíferos nativos de talla reducida y media no evidencian grandes variaciones de abundancia durante el período de estudio pero las de mayor tamaño redujeron sus poblaciones. El conejo europeo (Oryctolagus cuniculus) es una de las especies más abundantes del área, y estaría siendo favorecido por la modificación del paisaje. En la dieta del águila (Geranoaetus melanoleucus) y del peuco (Parabuteo unicinctus) se observó una disminución sostenida de la principal presa nativa, el degú (Octodon degus) y un aumento de la presa exótica, el conejo. Además se observó una tendencia a la disminución de la riqueza y abundancia de aves rapaces durante el período de estudio, la que se asociaría a la expansión urbana y al deterioro del matorral natural. Postulamos que la expansión urbana y la pérdida y fragmentación del matorral han producido una disminución de la población de degú, con su consecuente disminución en la dieta de águilas y peucos, lo que a su vez ha potenciado un incremento de depredación sobre conejo.
\end{abstract}

Palabras clave: ecología del paisaje, especies invasoras, especies nativas, tendencias temporales.

\begin{abstract}
San Carlos de Apoquindo, a piedemont area to the east of Santiago city, is a site with 30 years of data on vertebrates. Using LANDSAT satellite imagery for the years 1975, 1989 and 2003, we studied the spatiotemporal trajectory of land use and its putative effects on vegetation, small mammals and raptors. On the basis of a landscape-level analysis, we interpreted long-term trends in the abundance of small mammals and in the diet of raptors. Satellite imagery reveals an accelerated expansion of the urban area in detriment of agricultural land and native scrubland. Native mammal species of small and medium size do not display large variation of abundance during the 30 years study period, but larger-sized native mammals show reduced population numbers. The European rabbit (Oryctolagus cuniculus) currently is among the most abundant mammal species in the area, and seems to be favored by the temporal transition from dense to sparse scrubland. In the diets of Chilean Eagle (Geranoaetus melanoleucus) and of native Harris' Hawk (Parabuteo unicinctus) there is a marked decrease of the native rodent prey Octodon degus and a concomitant increase of the exotic prey Oryctolagus cuniculus. In addition, there is a decreasing trend in richness and abundance of raptors throughout the study period, which is associated to both urban expansion and scrubland fragmentation and reduction. We propose that urban encroachment, together with areal reduction and fragmentation of native scrubland, has resulted in a decrease of the Octodon degus population, and in its subsequent decrease in the diet of both native eagles and hawks, which in turn has determined increased predation on exotic rabbits.
\end{abstract}

Key words: invasive species, landscape ecology, native species, temporal trends. 


\section{INTRODUCCIÓN}

Las cinco regiones mediterráneas del mundo destacan por su rica biota y endemismos. Por su alto valor ambiental y fuerte presión antrópica, la región mediterránea en Chile central se considera como una de las 34 áreas críticas para la conservación de la biodiversidad del planeta (Myers et al. 2000, Conservation International 2007). Antiguamente, la vegetación natural de Chile central estaba dominada por bosques esclerófilos (Gajardo 1994), los que han sido degradados a la forma de matorrales fragmentados.

La ciudad de Santiago se ubica aproximadamente a $550 \mathrm{msnm}$, ocupa gran parte del valle de la Región Metropolitana, en una de las áreas mediterráneas más representativas de Chile (di Castri 1968) y en sus $15,800 \mathrm{~km}^{2}$ contiene una población de 6 millones de habitantes. El importante crecimiento poblacional y la ausencia de una planificación sustentable, han tenido como consecuencia una rápida y desordenada expansión de la ciudad hacia las estribaciones de la precordillera andina, alcanzando actualmente la cota de los $1,000 \mathrm{msnm}$, con pérdida de ambientes naturales y problemas ambientales tales como contaminación atmosférica por polvo, pérdida de suelos, inundaciones, remociones en masa y escasez de áreas verdes (Hajek et al. 1990, Romero et al. 1999, GORE-RM 2000¹).

A pesar de la rápida transformación del paisaje en Chile central, hay escasa evidencia de los impactos que este proceso ha generado sobre las poblaciones animales, aunque Romero et al. (1999) y Romero \& Órdenes (2004) señalaban que el rápido crecimiento urbano de la ciudad de Santiago ha tenido como consecuencia la contaminación de la atmósfera, del agua y de los suelos, la capacidad de infiltración y retención de humedad de los suelos, fragmentación de la vegetación e interrupción de corredores. A nivel nacional, otros estudios han revelado el importante efecto de los cambios del paisaje (hacia usos antrópicos) sobre la biodiversidad,

1 GORE-RM (2000) Proyecto de ordenamiento territorial ambientalmente sustentable para la Región Metropolitana. Primera etapa: Evaluación ambiental. Gobierno Regional de la Región Metropolitana, Universidad de Chile, Santiago. como ha sido el caso del área urbana de la ciudad de Concepción (Pauchard et al. 2006) y del Lago Budi (Peña-Cortés 2006), ambos estudios en el sur de Chile. Actualmente, se discute en el país sobre la asignación de categorías de conservación de flora y fauna, basadas fundamentalmente en el concepto de poblaciones y especies (Glade 1988, Núñez et al. 1997, Cofré \& Marquet 1999, Díaz-Páez \& Ortiz 2003), resultando frecuentemente en evaluaciones poblacionales subjetivas que no consideran la situación actual de los ambientes donde las especies ocurren.

En el marco de la evaluación de los potenciales efectos que puede generar la transformación del paisaje sobre los procesos que sustentan las poblaciones animales, en este estudio analizamos por una parte el cambio que ha experimentado el paisaje de la precordillera de Santiago durante un período de ca. 30 años, y por otro lado revisamos la trayectoria de las poblaciones de algunos micromamíferos y aves rapaces registrados en estudios de larga data en la localidad de San Carlos de Apoquindo en el piedemonte de la cordillera andina de Chile central.

\section{MÉTODOS}

\section{Área de estudio}

Para el análisis se definió arbitrariamente un área rectangular de 52,620 ha (que incluye a la localidad de San Carlos de Apoquindo) en el margen este del valle de Santiago (Fig. 1) en la Región Metropolitana de Chile. Ella incluye el sector oriente de la ciudad de Santiago y las áreas precordilleranas hasta los $2,000 \mathrm{msnm}$, teniendo como límites aproximados hacia el norte y sur los ríos Mapocho y Maipo respectivamente y centrado aproximadamente en $33^{\circ} 30^{\prime} \mathrm{S}$ y $70^{\circ} 30^{\prime} \mathrm{O}$. La vegetación natural del área está conformada por un matorral mediterráneo de tipo esclerófilo, que presenta menor cobertura en las laderas de exposición norte, donde se encuentra dominado por cactáceas y bromeliáceas, y en las zonas bajas, donde presenta una fisonomía de sabana dominada por espino Acacia caven (Mol.). En los fondos de quebrada y laderas de exposición sur se desarrolla el bosque esclerófilo (degradado a un matorral más denso o 
arborescente), con dominio de quillay Quillaja saponaria (Mol.), litre Lithrea caustica (Mol.), boldo Peumus boldus (Mol.) y peumo Criptocarya alba (Mol.). Históricamente, el área ha sufrido un proceso de degradación desde bosque esclerófilo hacia matorral abierto por acción combinada del fuego, talaje y pastoreo. En el último tiempo, el factor modelador principal del paisaje del área de estudio ha sido la expansión de la frontera urbana hacia el oriente.

\section{Evaluación espacial}

Para el análisis del desarrollo de la vegetación y uso del suelo en el área de estudio, se utilizaron imágenes satelitales LANDSAT de los años 1975, 1989 y 2003 (resolución de $79 \mathrm{~m}$ para la imagen de 1975 y $30 \mathrm{~m}$ para las restantes). Para el manejo de las imágenes, se procedió a su georreferenciación y a correcciones geométrica y radiométrica de cada una de las bandas. Los mapas temáticos fueron preparados $\mathrm{y}$ almacenados en un sistema de información geográfica mediante el uso de los software ERDAS IMAGINE 5.8 y ARCVIEW 3.2. Para los análisis espaciales se utilizó la extensión PATCH ANALYST (Elkie et al. 1999). Mediante una clasificación supervisada de las imágenes, se definieron cinco categorías de vegetación y uso del suelo para el área de estudio, a saber: urbano, agrícola, matorral abierto, matorral denso (correspondiente a matorrales arborescentes y bosques esclerófilos) y zonas sin vegetación (suelos desnudos y/o con nieves). Cada una de estas unidades fue caracterizada para los años 1975, 1989 y 2003 a escala de clase y paisaje, para lo que se calculó la superficie total de cada clase, el número de parches y tamaño medio de los parches como medidas de caracterización (McGarigal \& Marks 1994). La complejidad de las formas fue
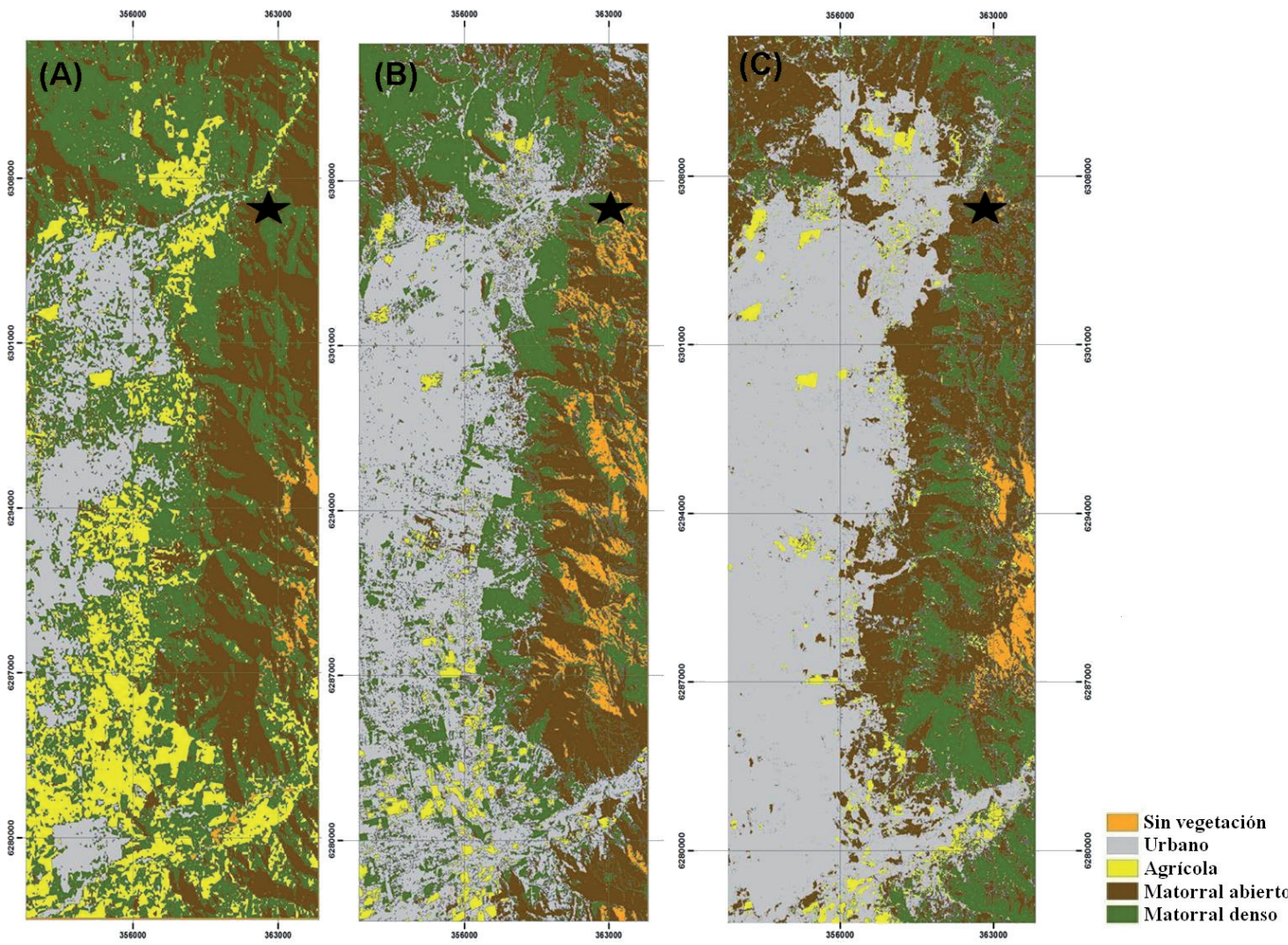

Fig. 1: Dinámica del paisaje en la precordillera de Santiago entre 1975 y 2003. A = 1975, B = 1989, $\mathrm{C}=2003$. La estrella muestra la localización de San Carlos de Apoquindo.

Landscape dynamics in the Santiago piedmont between 1975 and 2003. A = 1975, B = 1989, C = 2003. The star shows the location of San Carlos de Apoquindo. 
estimada a partir de la dimensión fractal (Mandelbrot 1984), indicador invariante de escala que permite interpretar la variación en la regularidad de las formas, en función de la relación área/perímetro.

\section{Micromamíferos}

Como sitio de referencia para el análisis de series históricas de datos sobre micromamíferos, se escogió el fundo San Carlos de Apoquindo (33 $23^{\prime} \mathrm{S} 70^{\circ} 31^{\prime} \mathrm{O}$; $1,000-2,000$ msnm), ubicado en el contrafuerte cordillerano del sector noreste de la cuenca de Santiago, donde se han realizado estudios de micromamíferos a partir de 1976. Para mayor información del sitio se puede consultar la revisión de Jaksic (2001). Se seleccionaron estudios realizados en 1976 (Jaksic \& Yáñez 1978), 1978 (Jaksic et al. 1981), 1981 (Simonetti 1983b) y 1984 (Iriarte et al. 1989a). El criterio de selección de los estudios fue la presencia de muestreos de tipo anual, pues entregan una mejor representación del ensamble de micromamíferos al documentar las variaciones intraanuales o estacionales (Jaksic \& Yáñez 1978, Jaksic et al. 1981, Iriarte et al. 1989a), y la presencia esporádica de especies (Simonetti 1983a, Iriarte \& Simonetti 1986).

Complementariamente, durante 2003 se realizaron capturas de micromamíferos en San Carlos de Apoquindo utilizando trampas Sherman $(8 \times 9 \times 24 \mathrm{~cm}$; H. B. Sherman Traps Inc., Tallahassee, FL, USA) cebadas con una mezcla de avena, atún y vainilla. El protocolo de capturas consideró muestreos durante las cuatro estaciones del año. Para poder contar con resultados comparables, se estandarizaron las capturas en términos porcentuales.

\section{Aves rapaces}

Se analizaron antecedentes publicados desde 1973 sobre presencia del roedor nativo degú (Octodon degus Molina, 1782) y del conejo europeo (Oryctolagus cuniculus Linnaeus, 1758) en egagrópilas de águila (Geranoaetus melanoleucus Vieillot, 1819) y de peuco (Parabuteo unicinctus Temminck, 1824), en el fundo San Carlos de Apoquindo y un área vecina denominada La Dehesa $\left(33^{\circ} 21^{\prime}\right.$ S $70^{\circ} 32^{\prime}$ $\mathrm{O}, 875 \mathrm{msnm})$. Para el análisis se consideró los siguientes estudios: para 1973, Schlatter et al.
(1980); para 1977, Yáñez \& Jaksic (1978); para 1979, Jaksic et al. (1980); para 1985, Jiménez \& Jaksic $(1989,1990,1993)$; y para 1988, Pavez et al. (1992). La información fue complementada con un análisis de egagrópilas (Marti et al. 2007) de peucos y águilas recolectadas durante 2006 en La Dehesa y San Carlos de Apoquindo.

Finalmente se realizó una estimación, de acuerdo a antecedentes de los autores (datos no publicados), respecto a la tendencia en la abundancia de algunas especies de aves rapaces en la precordillera de Santiago durante el período de estudio (véase Jaksic et al. 2001 para un esfuerzo similar).

\section{RESULTADOS}

\section{Dinámica del paisaje}

El análisis de las imágenes satelitales para los años 1975, 1983 y 2003 revela una expansión del área urbana, en desmedro de la vegetación natural y del uso del suelo agrícola principalmente en la mitad sur del área de estudio (Fig. 1). Los ambientes naturales fueron categorizados como matorral abierto y matorral denso (que en muchos casos corresponde a bosque degradado). Ambos tipos redujeron su superficie en toda el área de estudio (Fig. 2).

La pérdida de superficie del matorral ha sido acompañada por un notable aumento del número de parches, disminución del tamaño promedio de los parches y un aumento en la dimensión fractal (Fig. 3), todo lo cual denota reducción, fragmentación y deterioro en la calidad del matorral como hábitat para la fauna nativa. El mayor impacto sobre estos ambientes se produjo entre los años 1975 y 1989. Entre 1989 y 2003 el cambio ha sido menor, en parte por la restricción que ha tenido el crecimiento de la ciudad debido probablemente a factores físicos como la pendiente y factores limitantes de tipo legal (prohibición de construir sobre la cota $1,000 \mathrm{msnm}$ ).

\section{Micromamiferos}

Actualmente, el ensamble de micromamíferos en el área de estudio está conformado por ocho especies nativas, de las cuales cuatro tienen el carácter de endémicas. Especies como el degú 
de los matorrales (Octodon bridgesi Waterhouse, 1844) y el tunduco (Aconaemys fuscus Waterhouse, 1841) ocupaban Chile central en el holoceno tardío y aparentemente se extinguieron en esta área por causas antrópicas, tales como el desarrollo agrícola y ganadero (Saavedra \& Simonetti 2003).

Los datos de variación porcentual de las capturas de micromamíferos para el período de estudio (Fig. 4) deben ser interpretados con cautela, pues la composición de especies puede variar por factores puntuales tales como el número de días de muestreo y de trampas, o por factores globales y complejos como incursiones del fenómeno El Niño (Jaksic \& Lima 2003). No obstante, estos datos pueden entregar una aproximación a la tendencia en los cambios de abundancia en el periodo de estudio.

De las especies presentes en el área, el ratón colilargo (Oligoryzomys longicaudatus Bennett, 1832), el ratón lanudo (Abrothrix longipilis Waterhouse, 1837) y la yaca (Thylamys elegans Waterhouse, 1838) son colectados en mayor proporción en ambientes de matorral denso. De las especies exóticas invasoras, el conejo aparece poco representado debido al método de trampeo, pese a ser abundante (Miller \& Rottmann 1976, Jaksic et al. 1979), en tanto que el primer registro de un múrido invasor (Rattus rattus Linnaeus, 1758) fue documentado en 1981 (Simonetti 1983a) y a él se han sumado Mus musculus Linnaeus, 1758 y Rattus norvegicus Berkenhout, 1769 (Lobos et al. 2005). Es destacable la disminución de degú en las capturas a partir de 1978 y la baja representatividad histórica del ratón chinchilla (Abrocoma bennetti Waterhouse, 1837), correspondiendo estas dos especies a las de mayor tamaño en el área. El ratón orejudo de Darwin (Phyllotis darwini Waterhouse, 1837) y el ratón colilargo muestran una tendencia a aumentar su representatividad en las capturas.

La abundancia de las especies de talla pequeña y media no ha mostrado grandes variaciones en el período de estudio, en tanto que las de mayor tamaño han reducido o mantienen bajas representatividades en las colectas (Fig. 4), o incluso se extinguieron en tiempos históricos. Lo anterior coincide con el significativo incremento en el número de parches y la disminución del tamaño medio de los mismos, lo cual denota fragmentación del matorral esclerófilo de la precordillera de Santiago.

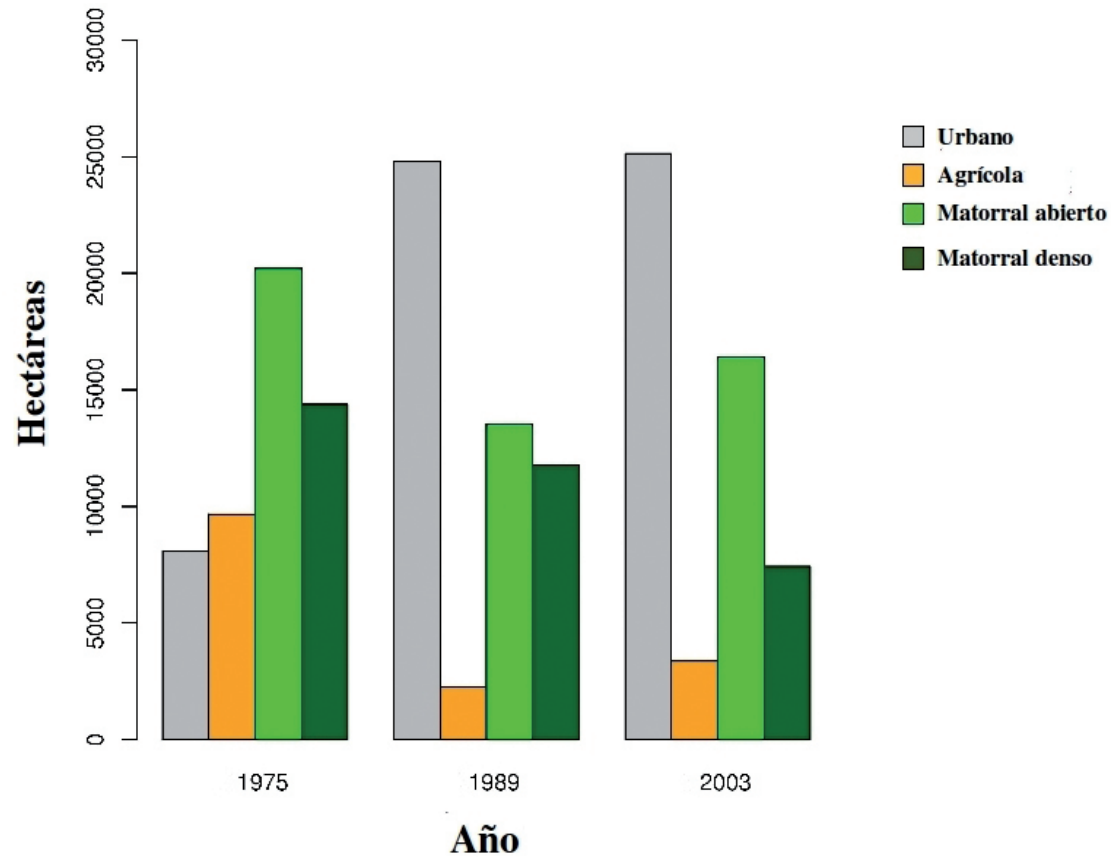

Fig. 2: Cambios en el uso de la tierra en la precordillera de Santiago entre 1975 y 2003.

Land use changes in the Santiago piedmont between 1975 and 2003. 


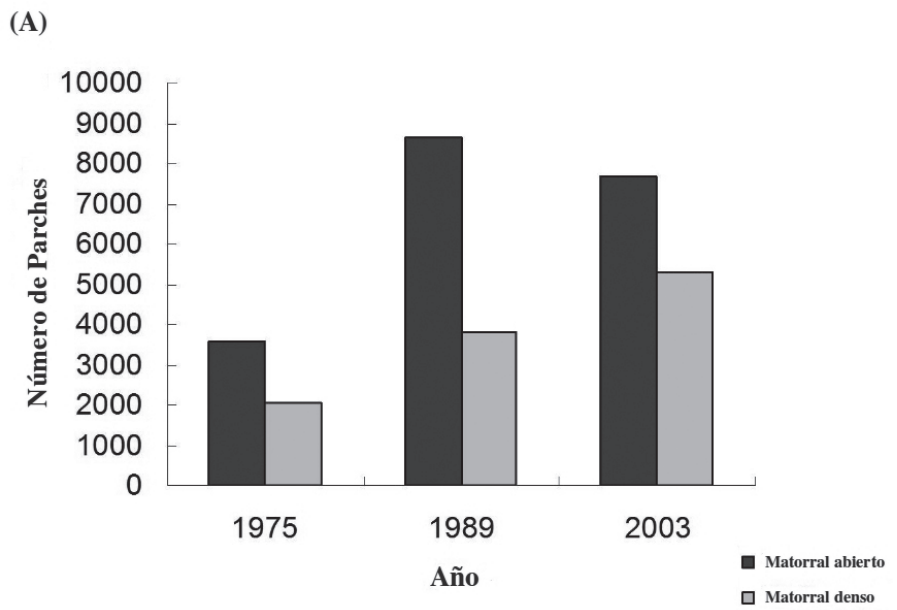

(B)

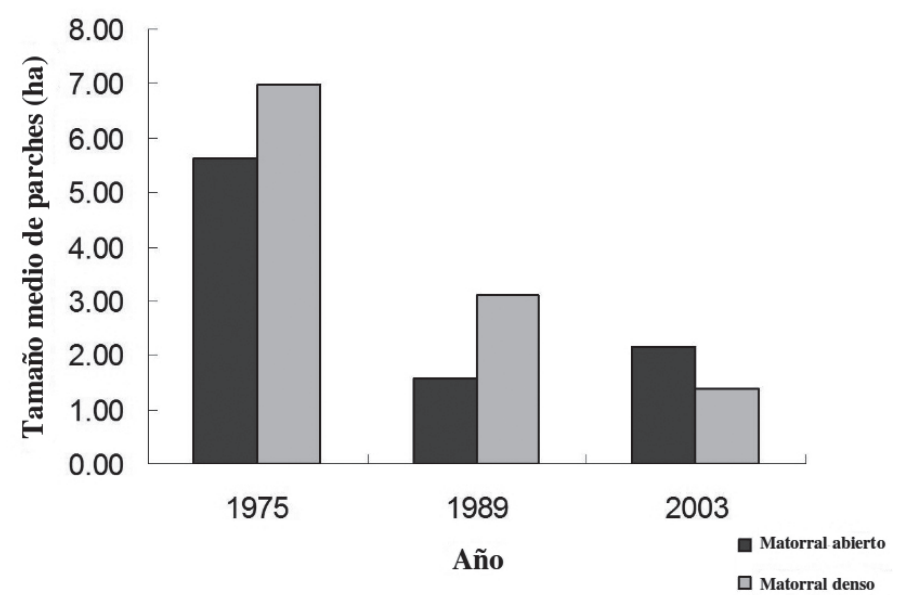

(C)

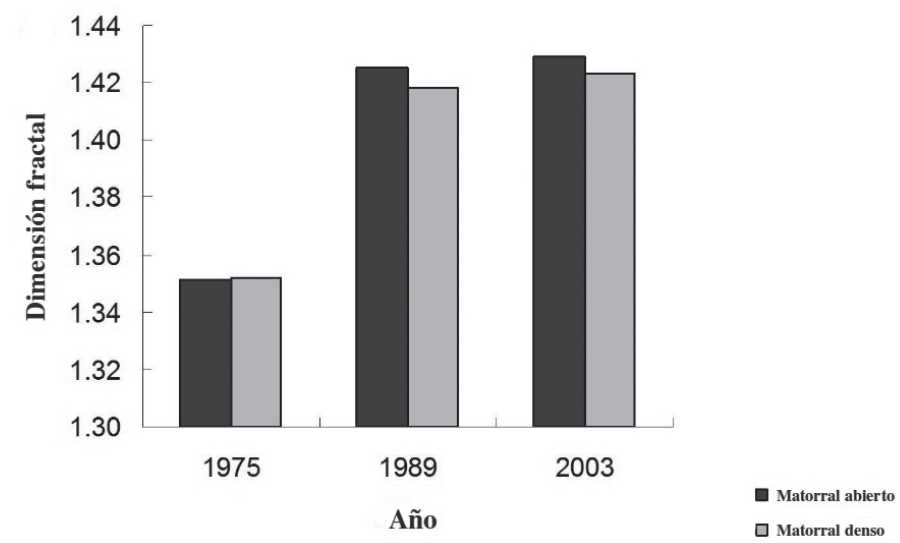

Fig. 3: Número de parches, tamaño medio de los parches (ha) y su dimensión fractal para matorral abierto y denso en la precordillera de Santiago entre 1975 y 2003.

Number of patches, mean size of patches (ha), and their fractal dimension for sparse and dense scrub in the Santiago piedmont between 1975 and 2003. 


\section{Aves rapaces}

Tres trabajos dan cuenta de la dieta del águila en San Carlos de Apoquindo entre 1973 y 1989. Schlatter et al. (1980) registraron en 1973-74 un $57.6 \%$ de degú y $18.8 \%$ de conejo $(n=170)$ en la dieta. En 1984-85, Jiménez \& Jaksic (1989) reportaron $32.3 \%$ de degú y $14.7 \%$ de conejo ( $\mathrm{n}=65)$. En 1987-89, Pavez et al. (1992) dieron cuenta de 18.9 \% de degú y 43.9 \% de conejo $(n=624)$. Finalmente, en 2006 nosotros obtuvimos $23.1 \%$ de degú y $48.7 \%$ de conejo (n =39). Por lo tanto, en la dieta de las águilas de San Carlos de Apoquindo se ha observado una clara tendencia a la disminución del degú (presa nativa) y al aumento del conejo (presa exótica), destacando el cambio registrado entre 1985 y 1988 (Fig. 5).

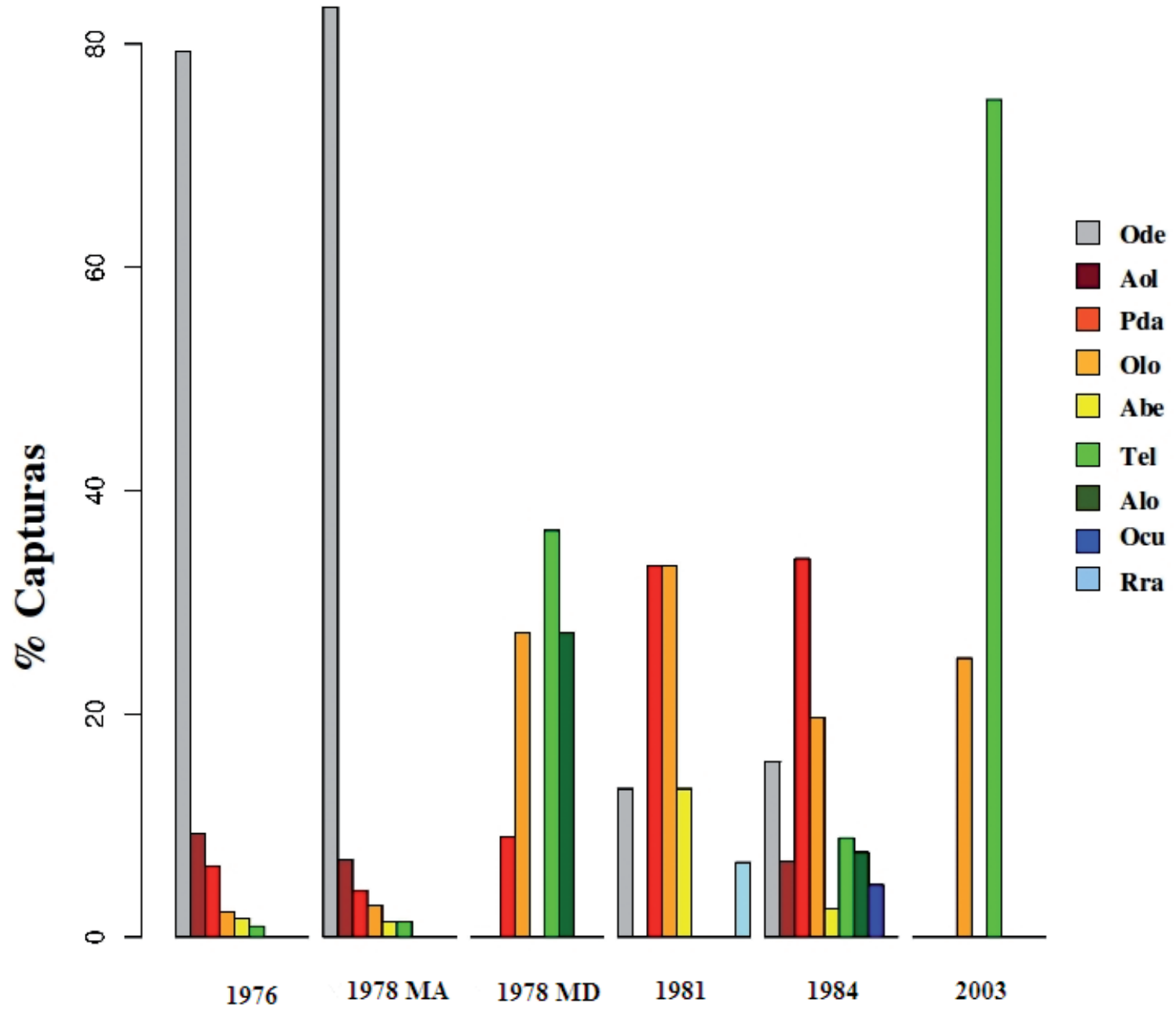

\section{Año del Estudio}

Fig. 4: Porcentaje de captura de micromamíferos en la precordillera de Santiago entre 1976 y 2003. Fuentes: para 1976, Jaksic \& Yáñez (1978); para 1978, Jaksic et al. (1981); para 1981, Simonetti (1983a, 1983b); para 1984, Iriarte et al. (1989a); para 2003, datos propios. Ode = Octodon degus, Aol = Abrothrix olivaceus, $\mathrm{Pda}=$ Pyllotis darwini, $\mathrm{Olo}=$ Oligoryzomys longicaudatus, $\mathrm{Abe}=$ Abrocoma bennetti, $\mathrm{Tel}=$ Thylamys elegans, $\mathrm{Alo}=$ Abrothrix longipilis, $\mathrm{Ocu}=$ Oryctolagus cuniculus, $\mathrm{Rra}=$ Rattus rattus $. \mathrm{MA}=$ matorral abierto $\mathrm{MD}=$ matorral denso.

Percent captures of small mammals in the Santiago piedmont between 1976 and 2003. Sources: for 1976, Jaksic \& Yañez (1978); for 1978, Jaksic et al. (1981); for 1981, Simonetti (1983a, 1983b); for 1984, Iriarte et al. (1989a); for 2003, authors' own data. Key to species same as above. MA = sparse scrub; MD = dense scrub. 
Para el caso del peuco, tres trabajos dan cuenta de su dieta entre 1976 y 1985 en San Carlos de Apoquindo y en La Dehesa. Yáñez \& Jaksic (1978) en 1976-78, encontraron 75.2 \% de degú, sin presencia de conejo $(\mathrm{n}=105)$. Para 1979 Jaksic et al. (1980) reportaron $64.5 \%$ de degú y $1.2 \%$ de conejo $(n=172)$. Para el período 1984-1985, Jiménez \& Jaksic (1993) documentaron $13.9 \%$ de degú y $18.2 \%$ de conejo $(n=165)$. Finalmente, durante 2006 nosotros obtuvimos ausencia total de degú y $84 \%$ de conejo en La Dehesa $(n=25)$. Por lo
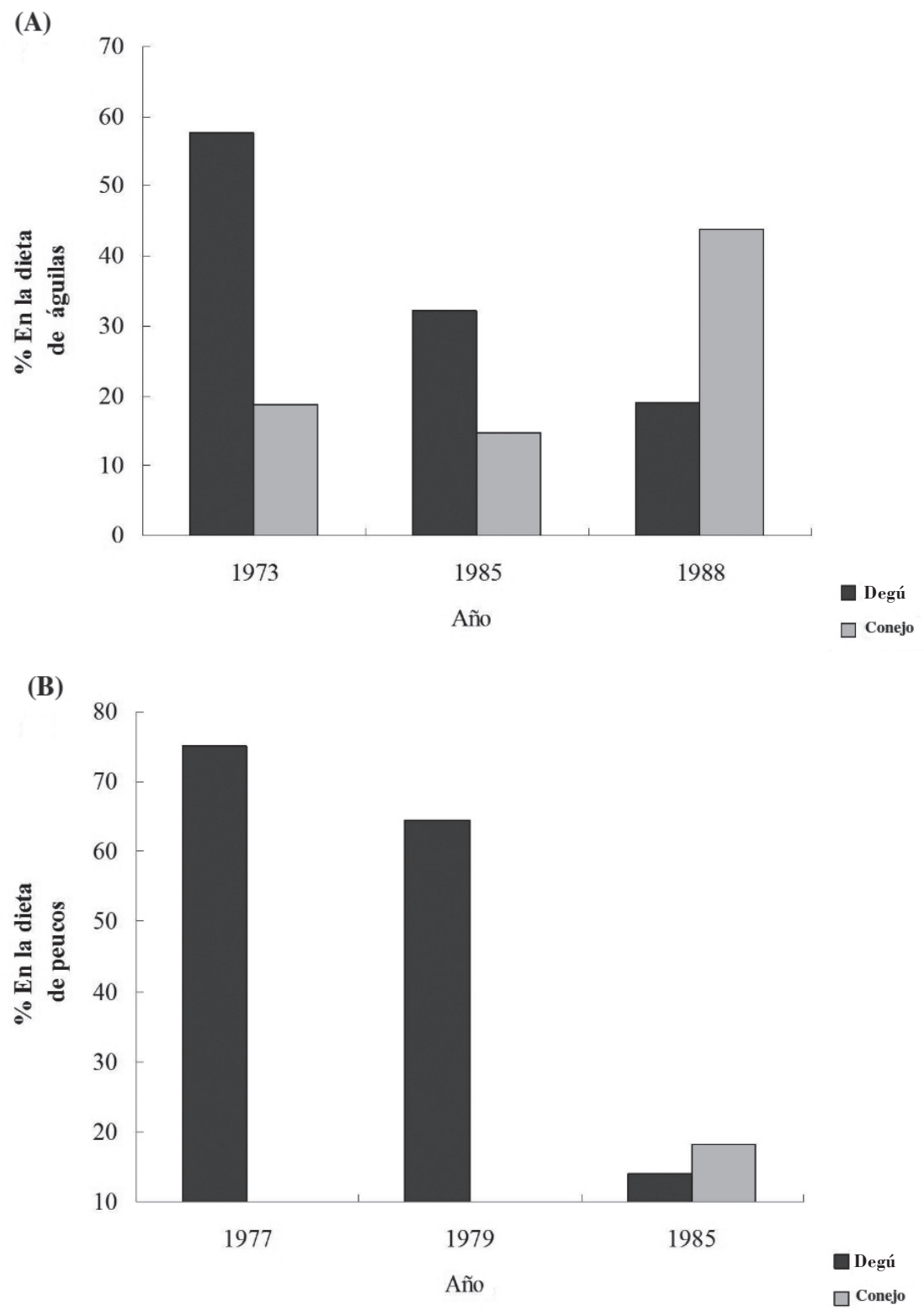

Fig. 5: Variación porcentual de degú nativo (Octodon degus) y conejo exótico (Oryctolagus cuniculus) en la dieta de águilas y peucos en la precordillera de Santiago entre 1977 y 2006 . Fuentes: para 1973, Schlatter et al. (1980); para 1977, Yáñez \& Jaksic (1978); para 1979, Jaksic et al. (1980); para 1985, Jiménez \& Jaksic (1989, 1990, 1993); para 1988, Pavez et al. (1992); para 2006, datos propios.

Percent occurrence of native degu (Octodon degus) and exotic rabbit (Oryctolagus cuniculus) in the diet of native eagles (A) and hawks (B) in the Santiago piedmont between 1977 and 2006. Sources: for 1973, Schlatter et al. (1980); for 1977, Yañez \& Jaksic (1978); for 1979, Jaksic et al. (1980); for 1985, Jiménez \& Jaksic (1989, 1990, 1993); for 1988, Pavez et al. (1992); for 2006, our own data. 
tanto, los datos indican que a partir de la década del los ochenta el peuco en la precordillera de Santiago disminuyó en forma abrupta el consumo de degú, al tiempo que incrementó la proporción de conejo (Fig. 5).

De las 24 especies de aves rapaces descritas por Jaksic et al. (2001) para la Región Metropolitana, 14 (58 \%) ocupan o han ocupado regularmente la precordillera de Santiago durante el período de estudio, observándose una tendencia a la disminución de su riqueza y abundancia, asociada al proceso de urbanización desde el valle hacia la precordillera. Espacialmente, se aprecia un aumento de la riqueza y abundancia de rapaces desde el área urbana hacia las zonas de matorral denso, pasando por una situación intermedia en áreas agrícolas, y alcanzando la máxima riqueza y abundancia en el matorral abierto.

Respecto a lo anterior cabe destacar la situación observada en el extremo noreste del área de estudio (San Carlos de Apoquindo y alrededores), donde a fines de la década de los ochenta, desaparecieron parejas establecidas de bailarín (Elanus leucurus Vieillot, 1818) y de nuco (Asio flammeus Pontoppidan, 1763), las que hasta principios de los ochenta eran comunes en el sector (EF Pavez, datos no publicados). Jaksic et al. (2001) citan a ambas especies como visitantes ocasionales en San Carlos de Apoquindo. El pequén (Athene cunicularia Molina, 1782) ha seguido la misma tendencia, y Jaksic et al. (2001) lo citan como residente actualmente escaso en San Carlos de Apoquindo. El cernícalo (Falco sparverius Linnaeus, 1758) y el tiuque (Milvago chimango Vieillot, 1816) son señalados por Jaksic et al. (2001) como comunes y abundantes, respectivamente, no obstante actualmente se observan en menor número que en las décadas de los setenta y ochenta (EF Pavez, datos no publicados).

Las especies de rapaces asociadas a quebradas y laderas de cerros menos accesibles y alejadas de urbanizaciones, han mantenido poblaciones más estables, particularmente el águila y el peuco. Pavez (2001) reportó para esos sectores de San Carlos de Apoquindo, la presencia de tres parejas de águilas residentes entre 1987 y 1989. Durante el verano 2006, solo dos territorios fueron ocupados, y el borde oeste del área ha sido abandonado, lo que coincide con la pérdida y deterioro de la zona baja de este sector debido a las urbanizaciones. Una situación similar se observa con el peuco, que al ser una especie asociada a zonas más bajas que el águila, ha perdido una proporción aún mayor de su hábitat original en el área de estudio.

\section{DISCUSIÓN}

La ocupación humana en las regiones mediterráneas ha estado asociada a cambios severos en la configuración del paisaje original, con profundas consecuencias sobre la biota (Arroyo 1999, Myers et al. 2000). De hecho, los sectores con menor accesibilidad han conservado parte importante de su biota original (Alados et al. 2004), pero la creciente demanda por nuevos territorios (para urbanización, cultivos, recreación) amenaza la integridad de estos espacios naturales (Pauchard et al. 2006).

La dinámica del paisaje en la precordillera de Chile central, muestra entre 1975 y 2003 un cambio importante en el uso del suelo, con una pérdida porcentual de $20.3 \%$ para los matorrales en su conjunto y de $12 \%$ para el uso agrícola. Solo el uso urbano muestra una ganancia de $31.5 \%$ entre ambas fechas, lo que demuestra el acelerado crecimiento de la urbanización del territorio. No obstante, ello representa solo una parte del histórico proceso de urbanización, pues de acuerdo a Romero et al. (1999), el máximo cambio en el uso de la tierra en esta área ocurrió entre los años 1962 y 1974, donde 7,544 ha cambiaron de uso agrícola a urbano. Diversos autores (Paul \& Meyer 2001, Riley et al. 2005, Mc Kiuney 2006, Pauchard et al. 2006) coinciden en que la urbanización es una de las principales amenazas a la biodiversidad. La mantención de áreas "tampón” tales como los sistemas agrícolas tradicionales, se señala como una importante herramienta en la conservación de la biodiversidad en sistemas mediterráneos de España (De Miguel 1999) y otros continentes (Mc Neely \& Schroth 2006).

Los espacios naturales de la precordillera de Chile central (matorrales y bosques esclerófilos) han sido fuertemente impactados en atributos tales como el número de parches, 
tamaño promedio de los parches y su grado de complejidad, lo cual denota un importante nivel de fragmentación y pérdida de hábitat (Peña-Cortés et al. 2006). En este contexto, la disminución del tamaño de los parches puede generar impactos importantes sobre las especies especialistas en hábitat (Franklin \& Forman 1987), lo que pudo haber ocurrido con el degú de los matorrales, posiblemente extinto en la precordillera de Santiago (Saavedra \& Simonetti 2003) y que puede estar ocurriendo con el degú, Octodon degus.

Por otra parte, la modificación del paisaje favorece la colonización por especies invasoras, las que a su vez alteran los atributos de los ecosistemas (Dukes \& Mooney 2004), generando en muchos casos nuevas invasiones. El conejo europeo ha invadido con éxito el piedemonte de Santiago, hecho que ha sido asociado fundamentalmente a la antigua baja depredación por sus posibles controladores naturales (Jaksic \& Soriguer 1981, Jaksic \& Ostfeld 1983). No obstante lo anterior, la transformación del paisaje también puede estar asociada a la colonización de esta especie. Ello resulta evidente en la invasión de los múridos exóticos en la región mediterránea de Chile central (Lobos et al. 2005). Del mismo modo, actividades productivas como la ganadería, han sido asociadas a la modificación del hábitat y su sinergia con especies invasoras tales como el conejo (Simonetti 1983b). La alta cobertura del matorral es importante para que los micromamíferos nativos eviten a sus predadores (Iriarte et al. 1989a, 1989b), en tanto que el conejo usa áreas con baja cobertura arbustiva, aparentemente más interesado en obtener forraje que en evitar predadores (Jaksic \& Soriguer 1981, Simonetti 1983b). Por lo tanto, la modificación del paisaje favorecería al conejo y no a los micromamíferos nativos.

En relación a la trayectoria temporal del ensamble de micromamíferos nativos del área, las especies que más han disminuido su representación en las capturas han sido las de mayor tamaño. Saavedra \& Simonetti (2003) señalan que en el holoceno tardío, la precordillera de Chile central era ocupada por el degú de los matorrales (especialista en matorral denso) y por el tunduco (fosorial), concluyendo que ambas especies fueron afectadas por las modificaciones del hábitat efectuadas por los humanos. El ratón chinchilla, especialista en matorral denso, está presente con baja representatividad en diversas zonas del área de estudio (GA Lobos, datos no publicados). Un caso destacable es el degú, roedor que generalmente se consideraba como muy abundante e incluso como plaga (Mann 1987), lo que denota su elevada abundancia histórica. No obstante ello, nuestra serie de tiempo evidencia una disminución importante de dicha especie en el área, y consideramos que dos son los principales factores asociados a dicha declinación. En primer lugar, la pérdida de superficie de matorral, acompañada por la fragmentación y deterioro en la calidad del matorral como hábitat para especies originarias. En segundo lugar, y aunque no se cuenta con información de carga animal ganadera, la potencial competencia por alimento y deterioro del suelo (erosión por caprinos, destrucción del suelo por grandes ungulados) puede ser otro factor de impacto.

La tendencia a la baja en la población de degú se correlaciona con su disminución en la dieta del águila y peuco, y con el aumento del conejo en la dieta de ambos predadores locales. Estudios de dieta de zorro (Pseudalopex culpaeus Molina, 1782) en San Carlos de Apoquindo muestran el mismo patrón, con $19.7 \%$ de conejo en 1976 (Jaksic et al. 1980), $37 \%$ en 1983 (Simonetti 1986) y $48 \%$ en 1984 (Iriarte et al. 1989b). E1 creciente consumo de conejo por el águila a través del tiempo, se ha descrito como una respuesta funcional frente a una presa relativamente más abundante (Pavez et al. 1992). Postulamos que este cambio relativo se ha producido por la importante disminución del degú en el área de estudio, que era la presa principal del águila y el peuco hasta mediados de los ochenta. Cabe destacar la coincidencia de este proceso con el importante deterioro del hábitat de matorral que se observa durante el período 1975-1989 en el área de estudio. Además, Pavez et al. (1992) afirman que el conejo se encuentra presente en San Carlos de Apoquindo desde antes de la década de 1940 y que su número no parece haber aumentado notoriamente durante los años previos a su estudio, lo cual estaría reforzando la idea de que su aumento en la dieta de águilas y peucos no se debería a 
un aumento en su abundancia sino que a una disminución de la oferta relativa de degú.

Los cambios del paisaje precordillerano no solo han tenido efecto dietario en las especies de aves rapaces oportunistas y con capacidad de acceder a recursos del tamaño del conejo, como el águila y el peuco, sino que además aparentemente han provocado una disminución de la abundancia de otras especies que, como el bailarín, nuco y pequén, están más asociadas a matorrales y áreas agrícolas particularmente de zonas más bajas y por ello más intervenidas. Otros factores de presión, tales como la caza ilegal de aves rapaces, no han sido evaluados en este trabajo, pero esta actividad ha operado históricamente en el área.

Otro ejemplo de impacto sobre ensambles de micromamíferos y sus predadores en los últimos 30 años por pérdida de ambientes naturales en la precordillera de Santiago, se da en la Reserva Nacional Río Clarillo, ubicada inmediatamente al sur de nuestra área de estudio, donde gran parte de las especies registradas se ubica bajo los 1,600 msnm, correspondiente a matorral esclerófilo, área que presenta la mayor carga de usos (Díaz et al. 2002). Ambas situaciones han sido consecuencia de frecuentes modificaciones a los planes reguladores para la Región Metropolitana, los que han favorecido el desarrollo inmobiliario e industrial en desmedro de las áreas agrícolas y ambientes naturales. Además, el área mediterránea de Chile central se encuentra escasamente representada en el Sistema Nacional de Áreas Silvestres Protegidas del Estado (Muñoz et al. 1996, Simonetti 1999, Pauchard \& Villarroel 2002).

Desde un punto de vista político-ambiental, la planificación del uso del territorio (Ordenamiento Territorial) es una herramienta legal que debiera ser implementada con urgencia en Chile y que debiera tener en cuenta los requerimientos ambientales para la conservación de poblaciones viables y el alto valor de los sistemas naturales de esta región mediterránea, una de las cinco en el mundo y uno de los 34 "hot spots" de biodiversidad en el planeta. Otro aspecto importante de enfatizar es la carencia de sitios con estudios de larga data en biodiversidad, lo que impide realizar análisis en escalas espacio-temporales mayores.
Finalmente, creemos necesario complementar nuestro estudio con investigaciones sobre otros factores que pudieran estar impactando los ensambles de micromamíferos nativos y sus predadores, tales como el fuego, interferencia por especies exóticas, la ganadería y la caza.

\section{MATERIAL COMPLEMENTARIO}

La versión en inglés de este artículo está disponible como Material Complementario online en http://rchn.biologiachile.cl/ suppmat/2010/1/MC_Pavez_et_al_2010.pdf

\section{AGRADECIMIENTOS}

Este trabajo fue posible en parte gracias al apoyo de sendas becas doctorales otorgadas a EF Pavez y GA Lobos por la Comisión Nacional de Investigación Científica y Tecnológica de Chile, CONICYT. También se agradece el apoyo de CF Estades y de la Facultad de Ciencias Forestales de la Universidad de Chile. Y finalmente a FONDECYT/FONDAP 1501-0001 y 1707-0004.

\section{LITERATURA CITADA}

ALADOS CL, Y PUEYO, O BARRANTES, J ESCOS, L GINER \& AB ROBLES (2004) Variations in landscape patterns and vegetation cover between 1957 and 1994 in a semiarid Mediterranean ecosystem. Landscape Ecology 19: 543-559.

ARROYO MTK (1999) Criterios e indicadores para la conservación de la biota de los ecosistemas mediterráneos. Revista Chilena de Historia Natural 72: 473-474.

COFRE H \& PA MARQUET (1999) Conservation status, rarity and geographic priorities for conservation of Chilean mammals: An assessment. Biological Conservation 88: 53-68.

CONSERVATION INTERNATIONAL (2007) Biodiversity hotspots. URL: http:// www.biodiversityhots pots.org/Pages / default.aspx (accedido Septiembre 18, 2009).

DE MIGUEL JS (1999) Naturaleza y configuración del paisaje agrosilvopastoral en la conservación de la diversidad biológica en España. Revista Chilena de Historia Natural 72: 547-557.

DÍAZ I, C SARMIENTO, L ULLOA, R MOREIRA, R NAVIA, E VÉLIZ \& C PEÑA (2002) Vertebrados terrestres de la Reserva Nacional Río Clarillo, Chile central: Representatividad y conservación. Revista Chilena de Historia Natural 75: 433-448.

DÍAZ-PÁEZ H \& JC ORTIZ (2003) Recategorización de la batracofauna de Chile. Revista Chilena de Historia Natural 76: 509-525. 
DI CASTRI F (1968) Equisse écologique du Chili.

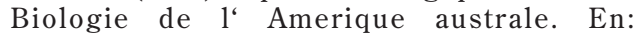
Debouteville $\mathrm{Cl} \&$ D Rapaport (eds) Editions du centre national de la Recherche Scientifique: 752. Paris, Francia.

DUKES JS \& HA MOONEY (2004) Disruption of ecosystem processes in western North America by invasive species. Revista Chilena de Historia Natural 77: 411-437.

ELKIE P, R REMPEL \& A CARR (1999) Patch analyst user's manual. Ontario Ministry of Natural Resources. Northwest Science \& Technology, Thunder Bay, Ontario, Canada.

FRANKLIN JF \& FORMAN RTT (1987) Creating landscape patterns by forest cutting: Ecological consequences and principles. Landscape Ecology 1: 5-18.

GAJARDO R (1994) La vegetación natural de Chile: Clasificación y distribución geográfica. Editorial Universitaria, Santiago, Chile.

GLADE A (1988) Libro rojo de los vertebrados terrestres de Chile. CONAF, Santiago, Chile.

HAJEK ER, P GROSS \& G ESPINOZA (1990) Problemas ambientales de Chile. AID - Pontificia Universidad Católica de Chile, Santiago, Chile.

IRIARTE A \& JA SIMONETTI (1986) Akodon andinus (Philippi, 1858): Visitante ocasional del matorral esclerófilo centro-chileno. Noticiario Mensual del Museo Nacional de Historia Natural (Chile) 311: 6-7.

IRIARTE JA, LC CONTRERAS \& FM JAKSIC (1989a) A long term study of a small mammal assemblage in the central Chilean matorral. Journal of Mammalogy 70: 79-87.

IRIARTE JA, JE JIMÉNEZ, LC CONTRERAS \& FM JAKSIC (1989b) Small mammal availability and consumption by the fox Dusicyon culpaeus, in central Chilean scrublands. Journal of Mammalogy 70: 641-645.

JAKSIC FM (2001) Patrones de variación espaciotemporal de plantas y animales en San Carlos de Apoquindo, Chile central. Revista Chilena de Historia Natural 74: 477-502.

JAKSIC FM \& M LIMA (2003) Myths and facts on ratadas: Bamboo blooms, rainfall peaks and rodent outbreaks in South America. Austral Ecology 29: 237-251.

JAKSIC FM \& RS OSTFELD (1983) Numerical and behavioral estimates of predation upon rabbits in mediterranean-type shrublands: A paradoxical case. Revista Chilena de Historia Natural 56: 3949.

JAKSIC FM \& RC SORIGUER (1981) Predation upon the European rabbit (Oryctolagus cuniculus) in mediterranean habitats of Chile and Spain: A comparative analysis. Journal of Animal Ecology 50: 269-281.

JAKSIC FM \& JL YAÑEZ (1978) Variación anual de la composición comunitaria de micromamíferos en Los Dominicos, Santiago. Noticiario Mensual del Museo Nacional de Historia Natural (Chile) 267: 10-11.

JAKSIC FM, ER FUENTES \& JL YAÑEZ (1979) Spatial distribution of the old world rabbit (Oryctolagus cuniculus) in central Chile. Journal of Mammalogy 60: 207-209.

JAKSIC FM, JL YAÑEZ \& ER FUENTES (1981) Assessing a small mammal community in central Chile. Journal of Mammalogy 62: 391-396.

JAKSIC FM, EF PAVEZ, JE JIMENEZ \& JC TORRES-
MURA (2001) The conservation status of raptors in the Metropolitan Region, Chile. Journal of Raptor Research 35: 151-158.

JIMÉNEZ JE \& FM JAKSIC (1989) Behavioral ecology of grey eagle-buzzards, Geranoaetus melanoleucus, in central Chile. Condor 91: 913221.

JIMÉNEZ JE \& FM JAKSIC (1990) Historia natural del águila Geranoaetus melanoleucus: Una revisión. El Hornero (Argentina) 13: 97-110.

JIMÉNEZ JE \& FM JAKSIC (1993) Observations on the comparative behavioral ecology of Harris' Hawk in central Chile. Journal of Raptor Research 27: 143-148.

LOBOS G, M FERRES \& RE PALMA (2005) Presencia de los géneros invasores Mus y Rattus en áreas naturales de Chile: Un riesgo ambiental y epidemiológico. Revista Chilena de Historia Natural 78: 113-124.

MANDELBROT B (1984) Les objects fractals Flamarion. Paris, Francia.

MANN G (1978) Los pequeños mamíferos de Chile. Gayana Zoología (Chile) 40: 1-342.

MARTI CD, M BECHARD \& FM JAKSIC (2007) Food habits. En: Bird DM \& KL Bildstein (eds) Raptor research and management techniques: 129-151. Hancock House Publishers, Blaine, Washington, USA.

MC GARIGAL K \& BJ MARKS (1994) FRAGSTATS: Spatial pattern analysis program for quantifying landscape structure. General Technical Report PNW-351. U.S. Department of Agriculture, Forest Service. Portland, OR.

MC KIUNEY ML (2006) Urbanization as a major cause of biotic homogenization. Biological Conservation 127: 247-260.

MC NEELY JA \& G SCHROTH (2006) Agroforestry and biodiversity conservation: Traditional practices, present dynamics and lessons for the future. Biodiversity and Conservation 15: 549554.

MILLER SD \& J ROTTMANN (1976) Guía para el reconocimiento de mamíferos chilenos. Editorial Gabriela Mistral, Santiago, Chile.

MUÑOZ M, H NÚÑEZ \& J YÁÑEZ (1996) Libro rojo de los sitios prioritarios para la conservación de la diversidad biológica en Chile. Corporación Nacional Forestal, Santiago, Chile.

MYERS N, R MITTERMEIR, CG MITTERMEIR, GA DA FONSECA \& J KENT (2000) Biodiversity hotspots for conservation priorities. Nature 403: 853-858.

NÚÑEZ H, V MALDONADO \& R PÉREZ (1997) Reunión de trabajo con especialistas de herpetología para categorización de especies según estados de conservación. Noticiario Mensual Museo Nacional de Historia Natural (Chile) 329: 12-19.

PAUCHARD A \& P VILLARROEL (2002) Protected areas in Chile: History, current status and challenges. Natural Areas Journal 22: 318-330.

PAUCHARD A, M AGUAYO, E PEÑA \& R URRUTIA (2006) Multiple effects of urbanization on the biodiversity of developing countries: The case of a fast-growing metropolitan area (Concepción, Chile). Biological Conservation 127: 272-281.

PAUL MJ \& JL MEYER (2001) Streams in the urban landscape. Annual Review of Ecology and Systematics 32: 333-365.

PAVEZ EF (2001) Biología reproductiva del águila 
(Geranoaetus melanoleucus) en Chile central. Revista Chilena de Historia Natural 74: 687-697.

PAVEZ EF, CA GONZÁLEZ \& JE JIMÉNEZ (1992)

Diet shifts of black-chested eagle (Geranoaetus melanoleucus) from native prey to European rabbits in Chile. Journal of Raptor Research 26: 27-32.

PEÑA-CORTÉS F, G REBOLLEDO, K HERMOSILLA, E HAUENSTEIN, C BERTRAN, R SCHLATTER \& J TAPIA (2006) Dinámica del paisaje para el período 1980-2004 en la cuenca costera del Lago Budi, Chile. Consideraciones para la conservación de sus humedales. Ecología Austral 16: 183-196.

RILEY SPD, GT BUSTEED, LB KATS, TL VANDERGON, LFS LEE et al. (2005) Effects of urbanization on the distribution and abundance of amphibians and invasive species in southern California streams. Conservation Biology 19: 1894-1907.

ROMERO H \& F ÓRDENES (2004) Emerging urbanization in the southern Andes. Environmental impacts of urban sprawl in Santiago de Chile on the Andean piedmont. Mountain Research and Development 24: 197201.

ROMERO H, M IHL, A RIVERA, P ZALAZAR \& SP
AZOCAR (1999) Rapid urban growth, land-use changes and air pollution in Chile. Atmospheric Environment 33: 4039-4048.

SAAVEDRA B \& JA SIMONETTI (2003) Holocene distribution of Octodontid rodents in central Chile. Revista Chilena de Historia Natural 76: 383-389.

SCHLATTER R, J YÁÑEZ \& FM JAKSIC (1980) Foodniche relationships between Chilean eagle and red-backed buzzard in central Chile. Auk 97: 897-898.

SIMONETTI JA (1983a) Occurrence of the black rat (Rattus rattus) in central Chile. Mammalia 47: 131-132.

SIMONETTI JA (1983b) Effect of goats upon native rodents and European rabbits in the Chilean matorral. Revista Chilena de Historia Natural 56: 27-30.

SIMONETTI JA (1986) Human-induced dietary shift in Dusicyon culpaeus. Mammalia 50: 406-408.

SIMONETTI JA (1999) Diversity and conservation of terrestrial vertebrates in mediterranean Chile. Revista Chilena de Historia Natural 72: 493-500.

YÁÑEZ J \& FM JAKSIC (1978) Presas de Parabuteo unicinctus en los alrededores de Santiago. Noticiario Mensual del Museo Nacional de Historia Natural (Chile) 23: 8-9. 
